\title{
Study on Spatial Mechanics Analysis Method of Excavator Attachment with Tiltrotator
}

\author{
Peng-Long HOU ${ }^{1}$, a , Feng-Yi LU1 ${ }^{1}$, Ge-Ning $\mathrm{XU}^{1}$ and Zhi-Ning MA ${ }^{1}$ \\ 1 Taiyuan University of science and technology, Department of mechanical engineering, China \\ a houpenglongsx@foxmail.com
}

Keywords: Excavator; Attachments; Spatial force.

\begin{abstract}
Usually a tiltrotator is mounted on the excavator such that the bucket of excavator can not only move in the plane of the excavator attachment, but also deviate from the plane. However, this generated additional bending moment and torque, and what was more, the traditional design method, which is used to design the excavator attachment without considering the tiltrotator, based on the condition that the bucket moved in the plane (in two dimensions) of the attachment is no longer suitable for the excavator with tiltrotator. In order to solve this problem, according to the equilibrium equations of spatial (in three dimensions) force system and matrix theory, linkage groups of excavator attachment with tiltrotator have been decomposed and analysed with the spatial static load. Through theoretical derivation, a suitable calculation method and a calculation process, which can be applied to the analysis of the spatial force, are obtained to solve the problem of the force analysis of the different parts of the excavator attachment with tiltrotator. Then the calculation results, which based on the calculation method in this paper, of bucket hinge points are compared with the results of the classical force analysis in the plane. The results show that analysis results based on the calculation method in this paper are not only correct, but also compatible with the plane force analysis.
\end{abstract}

\section{Introduction}

Ordinary excavator buckets can only excavate in a plane (in two dimensions). When the complex job needs to adjust the machine position frequently, work efficiency is very low. In addition, the function of excavator is single. When changing other work tools, such as breaker, grapple, and auger, the replacement process is complex and time-consuming. Some excavators are equipped with a quick coupler [1-2], for simplified attachments mounting, drama-tidally increasing the machine's utilization on the jobsite and saving the replacement of equipment time. Sometimes a tiltrotator is installed between the bucket and the arm such that the excavator bucket can be rotated through 360 degrees and tilted $+/-45$ degrees, in order to increase the flexibility and precision of the excavator.

After installing the tiltrotator, the bucket of excavator can not only move in the plane of the excavator attachment, but also deviate from the plane. However, this generated additional bending moment and torque, and what was more, the traditional design method, which assumed that the excavator attachment is a planar mechanism, composed of a boom, an arm, and a bucket, based on the condition that the bucket move in the plane of the excavator attachment is no longer suitable for the excavator attachment with tiltrotator [3]. In traditional design method of excavator, influence of the additional bending moment and torque, generated by special conditions, is discussed by Shi Qinglu [1]. Liu Chunli presented a finite element analysis study for a tiltrotator [2]. However, they neither considered the influence of the additional bending moment and torque, generating by tiltrotator, in their study. These traditional design methods, which are used to design the excavator attachment without considering the tiltrotator, based on the condition that the bucket moved in the plane (in two dimensions) of the attachment are no longer suitable for the excavator with tiltrotator.

In this paper, considering the influence of tiltrotator that may produce extra torque and moment of working equipment, using spatial mechanics analysis theory, a suitable calculation method and a calculation process which can be applied to the analysis of the spatial force are obtained to solve the problem of the force analysis of the different parts of the excavator attachment with tiltrotator. In addition, linkage groups of excavator attachment with tiltrotator have been decomposed, so that each 
group was acted by a similar force system. Finally, the approach, which is simplified and convenient to write program, to deal with spatial mechanics analysis method of excavator attachment with tiltrotator has achieved.

\section{Theoretical Foundation and Symbol Description}

\section{Theoretical Foundation}

The theoretical foundation of this paper is the theory of statics of the rigid bodies and systems of rigid bodies in [4], involving the equilibrium of rigid body and systems of rigid bodies in planar force system and spatial force system.

In planar problem (in two dimensions) when the body is acted by a system of coplanar system of forces, the scalar conditions of equilibrium are:

$$
\begin{gathered}
\sum F_{x}=0, \sum F_{y}=0, \\
\sum M_{Q}(\boldsymbol{F})=0,
\end{gathered}
$$

The scalar conditions of equilibrium of the rigid body that is acted by a system of optical system (in three dimensions) of forces are:

$$
\begin{gathered}
\sum F_{x}=0, \sum F_{y}=0, \sum F_{z}=0 \\
\sum M_{x}(\boldsymbol{F})=0, \sum M_{y}(\boldsymbol{F})=0, \sum M_{z}(\boldsymbol{F})=0
\end{gathered}
$$

To the same force system, the relationship between the resultant moments about the three reference axes and the resultant moments about the origin of the system of reference is:

$$
\begin{gathered}
{\left[\boldsymbol{M}_{o}(\boldsymbol{F})\right]_{x}=M_{x}(\boldsymbol{F})=y F_{z}-z F_{y}} \\
{\left[\boldsymbol{M}_{o}(\boldsymbol{F})\right]_{y}=M_{y}(\boldsymbol{F})=z F_{x}-x F_{z}} \\
{\left[\boldsymbol{M}_{o}(\boldsymbol{F})\right]_{z}=M_{z}(\boldsymbol{F})=x F_{y}-y F_{x}}
\end{gathered}
$$

The moment of a force about a point is:

$$
\boldsymbol{M}_{o}(\boldsymbol{F})=\boldsymbol{r} \times \boldsymbol{F}
$$

\section{Labelling Conventions and Illustration}

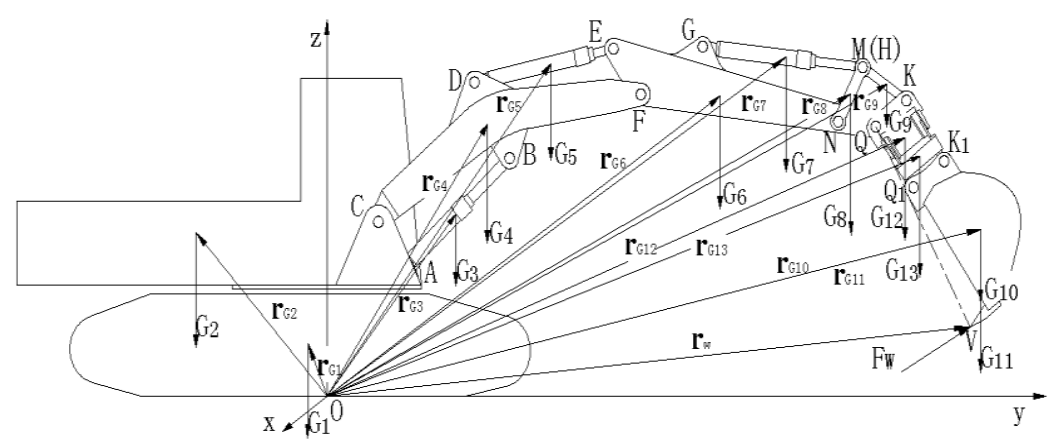

Figure 1. Labels and vector diagrams about an excavator

Figure 1 illustrates the component structures and loads as used throughout this report. The labels in the figure $r_{1} \sim r_{13}$ show the position vector of centres of gravity on the generalized coordinate system. The labels in figure $\boldsymbol{G}_{1} \sim \boldsymbol{G}_{13}$ show the gravity vector of the each component of the excavator. In figure $1, \boldsymbol{r}_{\mathrm{w}}$ presents the position vector of digging force and $\boldsymbol{F}_{\mathrm{w}}$ presents the digging force of excavator (the force always is tangent to the trajectory of bucket tip). Note that relevant points in the mechanism have been labelled with letters A-Q for the joint relevant to the analysis. 


\section{Static Force Analysis of Parts}

According to the structural features of the excavator attachment, there are three joint points, which are point $\mathrm{C}$, point $\mathrm{F}$ and point $\mathrm{Q}$, acted by a spatial force system [1]. Other parts and points are acted by a coplanar force system. The regularity of static force analysis of excavator attachment can be obtained as follows: when you calculate the forces on the parts of excavator from the bucket to the boom, excavator attachment can be decomposed into four linkage groups [5].
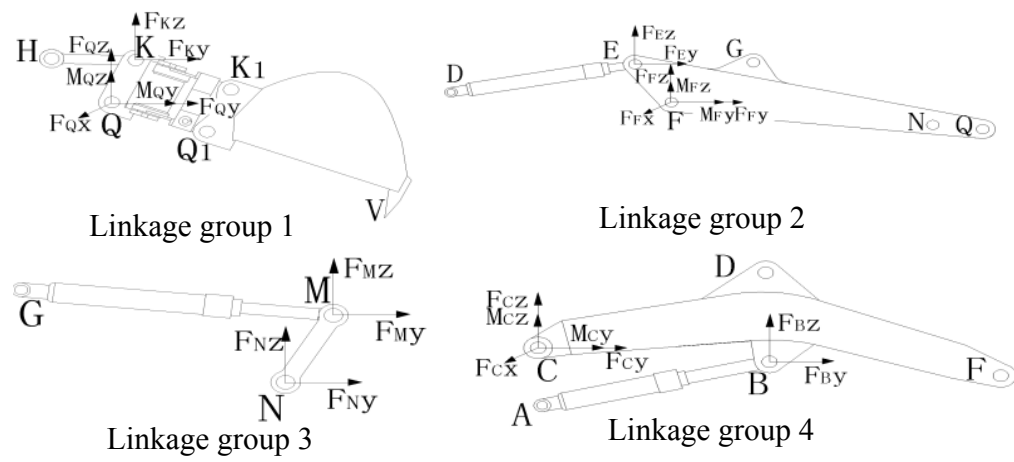

Figure 2. Four linkage groups

As shown in Figure 2, each group is made up of two parts - part of links (link, bucket hydraulic cylinder, boom hydraulic cylinders and arm hydraulic cylinders), and part of the key components (bucket, joystick, boom and arm) — and set of key components and links are connected by a planar hinge. The force acted at each linkage group is similar, that is, the links are in equilibrium of three forces (included gravity), and the force in the end of each link is unknown. Key components are in equilibrium of spatial force system. The forces at the points where key components and links are connected are unknown, and the forces at the points where key components and key components are connected also are unknown.

The solution is as follows: the forces at the point that a planar hinge connects the key components and links can be decomposed in the horizontal (X) and the vertical (Y) directions. Considering the parts of the linkage group is in equilibrium, $\Sigma \boldsymbol{M}=\mathbf{0}$, and taking moment about the hinge point of each part, two equations were obtained and the magnitudes of the unknown forces at the point that the key components and links are connected by a planar hinge can be computed from those two equations. In addition, the force at the point that the key components and key components are connected by a spatial hinge is unknown. The spatial hinge removes five degrees of freedom and these points bore bending and torsion. According to the theorem of the equilibrium of component parts, the equations 3 and 4 were obtained. The magnitudes of the unknown forces at the point that the key components and key components are connected by a spatial hinge can be computed from those equations. Following is an example of the linkage group 1 to introduce the computing method above.

\section{Static Force Analysis of Linkage Group 1}

Figure 3 illustrates the static forces acting at the bucket and tiltrotator.

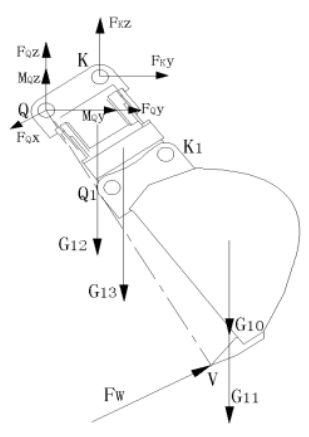

Figure 3. Free body diagrams of tiltrotator and bucket 


\section{Calculation of the Force at Point K}

Considering the bucket in equilibrium, $\Sigma \boldsymbol{M}=\mathbf{0}$, and taking moment about the boom to bucket hinge point Q leads to:

$$
\sum M_{Q \mathrm{x}}=M_{Q x}\left(\boldsymbol{F}_{\mathrm{k}}\right)+M_{Q x}\left(\boldsymbol{F}_{\mathrm{w}}\right)+\sum_{n=10}^{13} M_{Q x}\left(\boldsymbol{G}_{i}\right)=0
$$

Where $\boldsymbol{F}_{\mathrm{k}}$ is the force acting at tiltrotator hinge point $\mathrm{K}, \boldsymbol{F}_{\mathrm{w}}$ is the digging force.

The moment of force $\boldsymbol{F}_{\mathrm{k}}$ about point $\mathrm{Q}$ is:

$$
\boldsymbol{M}_{Q}\left(\boldsymbol{F}_{K}\right)=\boldsymbol{r}_{Q K} \times \boldsymbol{F}_{K}
$$

Where $\boldsymbol{r}_{Q K}$ is the relative position vector of $\mathrm{K}$ to $\mathrm{Q}$.

$$
\begin{gathered}
\boldsymbol{r}_{Q K}=\overline{Q K}=\left(\begin{array}{c}
x_{Q K} \\
y_{Q K} \\
z_{Q K}
\end{array}\right), \boldsymbol{F}_{K}=\left(\begin{array}{c}
F_{K x} \\
F_{K y} \\
F_{K z}
\end{array}\right) \\
\boldsymbol{M}_{Q}\left(\boldsymbol{F}_{K}\right)=\left(\begin{array}{l}
F_{K z} y_{Q K}-F_{K y} z_{Q K} \\
F_{K x} z_{Q K}-F_{K z} x_{Q K} \\
F_{K y} x_{Q K}-F_{K x} y_{Q K}
\end{array}\right)
\end{gathered}
$$

According to the structural characteristics of excavator attachment, limited to the arm, boom and bucket, which are acted by spatial force system, other parts are acted by planar force system. So equation 11 can be reduced as:

$$
\boldsymbol{M}_{Q}\left(\boldsymbol{F}_{K}\right)=\left(\begin{array}{c}
F_{K z} y_{Q K}-F_{K y} z_{Q K} \\
0 \\
0
\end{array}\right)
$$

The moment of force $\boldsymbol{F}_{\mathrm{w}}$ about point $\mathrm{Q}$ is:

$$
\boldsymbol{M}_{Q}\left(\boldsymbol{F}_{\mathrm{w}}\right)=\left(\begin{array}{l}
F_{w z} y_{Q V}-F_{w y} z_{Q V} \\
F_{w x} z_{Q V}-F_{w z} x_{Q V} \\
F_{w y} x_{Q V}-F_{w x} y_{Q V}
\end{array}\right)
$$

The moment of gravity of all parts about point $\mathrm{Q}$ is:

$$
\boldsymbol{M}_{Q}\left(\boldsymbol{G}_{i}\right)=\boldsymbol{r}_{Q i} \times \boldsymbol{G}_{i}
$$

Where $\boldsymbol{M}_{Q}\left(\boldsymbol{G}_{i}\right)$ is the moment of gravity of all parts about point Q; $\boldsymbol{r}_{Q i}$ is the relative position vector of the centres of Gravity to Q; $\boldsymbol{G}_{i}$ is the gravity vector of the each component of the excavator.

$$
\begin{gathered}
\boldsymbol{r}_{Q i}=\left(\begin{array}{c}
x_{Q i} \\
y_{Q i} \\
z_{Q i}
\end{array}\right), \boldsymbol{G}_{i}=G_{i} *\left(\begin{array}{c}
0 \\
0 \\
-1
\end{array}\right) \\
\boldsymbol{M}_{Q}\left(\boldsymbol{G}_{i}\right)=\left(\begin{array}{c}
-G_{i} y_{Q i} \\
-G_{i} x_{Q i} \\
0
\end{array}\right)
\end{gathered}
$$

Equation 9 can be rewritten by using the theory of equation 5:

$$
\left[\boldsymbol{M}_{Q}\left(\boldsymbol{F}_{\mathrm{k}}\right)\right]_{x}+\left[\boldsymbol{M}_{Q}\left(\boldsymbol{F}_{\mathrm{w}}\right)\right]_{x}+\sum_{n=10}^{13}\left[\boldsymbol{M}_{Q}\left(\boldsymbol{G}_{i}\right)\right]_{x}=0
$$

Substituting equations 12,13 and 15 into equation 16 yields: 


$$
F_{K z} y_{Q K}-F_{K y} z_{Q K}+F_{w z} y_{Q V}-F_{w y} z_{Q V}-\sum_{n=10}^{13} G_{i} y_{Q i}=0
$$

The second step: static force analysis of the link HK.

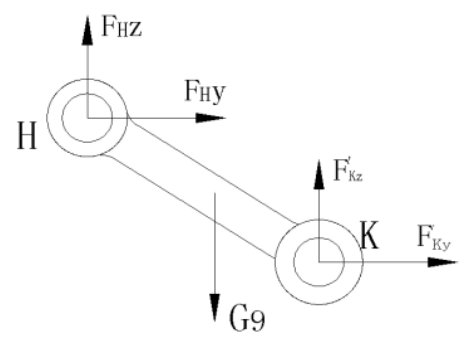

Figure 4. Free body diagrams of the link HK

Figure 4 illustrates the link acted by a planar force system. Static forces on points $\mathrm{H}$ and $\mathrm{K}$ can be calculated by considering the summation of forces must be equal to zero and summation of moments equal to zero for equilibrium condition of the link. Firstly, the reaction force acting on the joint points (at point $\mathrm{H}$ and $\mathrm{K}$ ) is resolved in the horizontal $(\mathrm{X})$ and the vertical $(\mathrm{Y})$ directions. Then considering the link in equilibrium $\sum M=0$, taking moment about the hinge point $\mathrm{H}$ leads to:

$$
\sum M_{H \mathrm{x}}=\left[\boldsymbol{M}_{H}\left(\boldsymbol{F}_{K ?}^{\prime}\right)\right]_{x}+\left[\boldsymbol{M}_{H}\left(\boldsymbol{G}_{9}\right)\right]_{x}=0
$$

Where $\boldsymbol{F}_{K}^{\prime}$ is the force in the joint $\mathrm{K}$ of the link, which is the reaction force in the link system, is equal to the given force $\boldsymbol{F}_{K}$ in the bucket system in value, but is opposed in direction.

$$
\begin{gathered}
\boldsymbol{M}_{H}\left(\boldsymbol{F}_{K}^{\prime}\right)=\boldsymbol{r}_{H K} \times \boldsymbol{F}_{K}^{\prime} \\
\boldsymbol{r}_{H K}=\overline{H K}=\left(\begin{array}{c}
x_{H K} \\
y_{H K} \\
z_{H K}
\end{array}\right), \boldsymbol{F}_{K}^{\prime}=\left(\begin{array}{l}
-F_{K x} \\
-F_{K y} \\
-F_{K z}
\end{array}\right)
\end{gathered}
$$

Conceding the link is acted by a planar force system. So equation 19 can be reduced:

$$
\boldsymbol{M}_{H}\left(\boldsymbol{F}_{K}^{\prime}\right)=-\left(\begin{array}{c}
F_{K z} y_{H K}-F_{K y} z_{H K} \\
0 \\
0
\end{array}\right)
$$

Substituting equation 20 into equation 18 yields:

$$
F_{K y} z_{H K}-F_{K z} y_{H K}-G_{9} y_{H 9}=0
$$

The magnitudes of the unknown forces $\boldsymbol{F}_{K y}$ and $\boldsymbol{F}_{K z}$ can be calculated from equations 17 and 21:

$$
\begin{gathered}
F_{K y}=\frac{\left(F_{w y} z_{Q V}-F_{w z} y_{Q V}+\sum_{n=10}^{13} G_{i} y_{Q i}\right) y_{H K}+G_{9} y_{H 9} y_{Q K}}{z_{H K} y_{Q K}-z_{Q K} y_{H K}} \\
F_{K z}=\frac{\left(F_{w y} z_{Q V}-F_{w z} y_{Q V}+\sum_{n=10}^{13} G_{i} y_{Q i}\right) z_{H K}+G_{9} y_{H 9} z_{Q K}}{z_{H K} y_{Q K}-z_{Q K} y_{H K}}
\end{gathered}
$$

Calculation of the Force at Point $H$

The scalar conditions of equilibrium of the link HK are:

$$
\sum F_{\mathrm{z}}=F_{K z}^{\prime}+F_{H z}+G_{9 z}=0
$$




$$
\sum F_{\mathrm{y}}=F_{K y}^{\prime}+F_{H y}=0
$$

From equations 24 and 25, the magnitudes of the unknown forces $\boldsymbol{F}_{H z}$ and $\boldsymbol{F}_{H y}$ can be calculated:

$$
\begin{gathered}
F_{H z}=\frac{\left(F_{w y} z_{Q V}-F_{w z} y_{Q V}+\sum_{n=10}^{13} G_{i} y_{Q i}\right) z_{H K}+G_{9} y_{H 9} z_{Q K}}{z_{H K} y_{Q K}-z_{Q K} y_{H K}}+G_{9} \\
F_{H y}=\frac{\left(F_{w y} z_{Q V}-F_{w z} y_{Q V}+\sum_{n=10}^{13} G_{i} y_{Q i}\right) y_{H K}+G_{9} y_{H 9} y_{Q K}}{z_{H K} y_{Q K}-z_{Q K} y_{H K}}
\end{gathered}
$$

\section{Calculation of the Force at Point $Q$}

Taking into account this object of study is acted by a spatial force system, the scalar conditions of equilibrium are obtained by equations 3 and 4 :

$$
\begin{gathered}
\sum F_{\mathrm{z}}=F_{Q z}+F_{K z}+F_{w z}+\sum_{n=10}^{13} G_{i z}=0 \\
\sum F_{\mathrm{y}}=F_{Q y}+F_{K y}+F_{w y}=0 \\
\sum F_{\mathrm{x}}=F_{Q x}+F_{w x}=0 \\
\sum M_{Q \mathrm{y}}=M_{Q \mathrm{y}}+M_{Q y}\left(\boldsymbol{F}_{\mathrm{w}}\right)+\sum_{n=10}^{13} M_{Q y}\left(\boldsymbol{G}_{i}\right)=0 \\
\sum M_{Q z}=M_{Q z}+M_{Q z}\left(\boldsymbol{F}_{\mathrm{w}}\right)=0
\end{gathered}
$$

From equations $28 \sim 32$, the magnitudes of the unknown forces at the point $\mathrm{Q}$ can be calculated:

$$
\begin{gathered}
F_{Q z}=-\left(F_{w z}+F_{K z}-\sum_{n=10}^{13} G_{i}\right) \\
F_{Q y}=-\left(F_{K y}+F_{w y}\right) \\
F_{Q x}=-F_{w x} \\
M_{Q \mathrm{y}}=F_{w z} x_{Q V}-F_{w x} z_{Q V}-\sum_{n=10}^{13} G_{i} x_{Q i} \\
M_{Q z}=F_{w x} y_{Q V}-F_{w y} x_{Q V}
\end{gathered}
$$

\section{Static Force Analysis of the Rest Linkage Group}

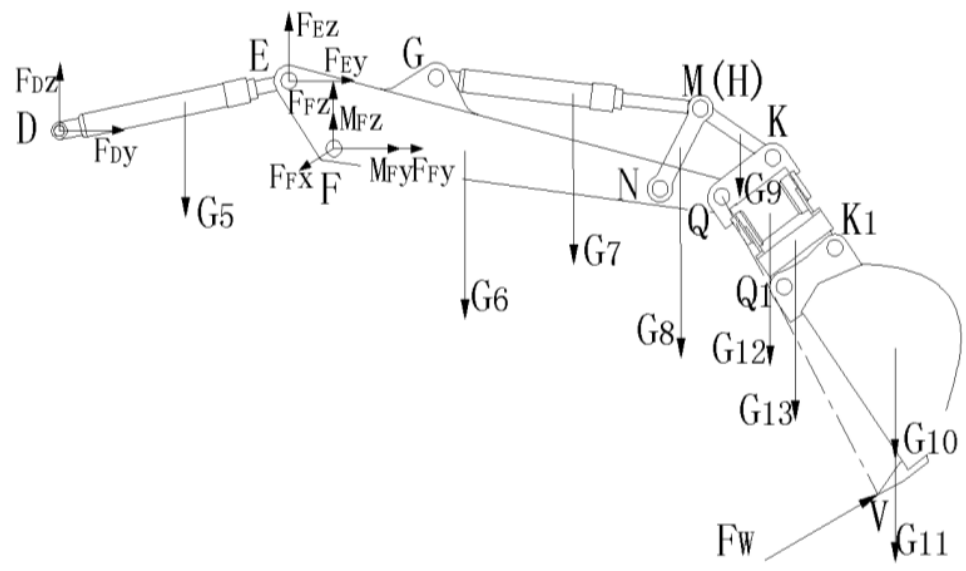

Figure 5. The linkage group of the arm 


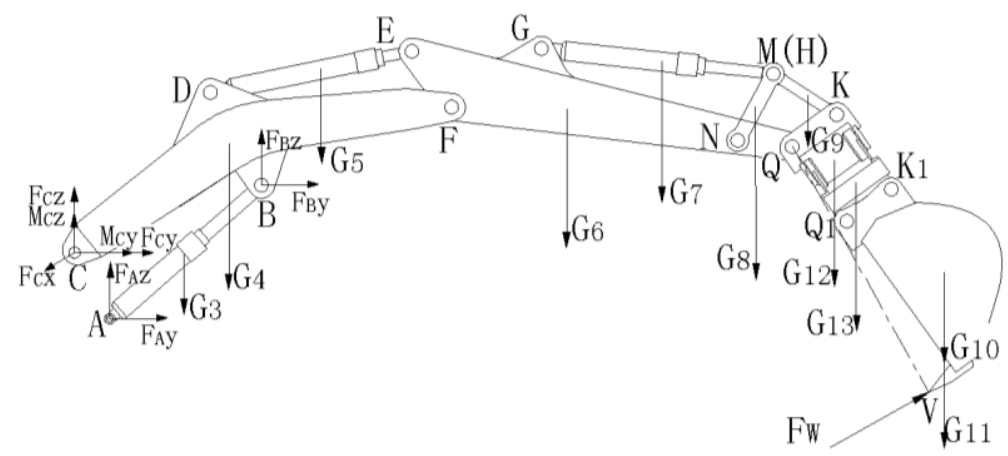

Figure 6. The linkage group of the boom

Using the above computing method of the forces at the hinge points of the group 1 can be determined, and the forces of the other linkage groups at hinge points can be obtained using the same steps. When determined the forces at the points of the arm linkage group and boom linkage, the new linkage group can be used as Figure 5, Figure 6. Moreover, the benefit of this is avoiding the internal reaction forces from the connections of the body.

\section{Comparison of Spatial and Planar Results of Static Force Analysis}

In this section, the spatial force analysis method that is presented in this paper will be illustrated by comparing the force analysis result which computed by the classical method which based on the assumption that the excavator was acted by a planar force system. Force acted at each linkage group is similar, so that just illustrated one group is enough.

\section{Comparison of the Force at Point $\mathrm{K}$}

Bucket penetration into a material is achieved by the digging force generated by the arm cylinder, and the digging force generated by the bucket cylinder [6-7]. This paper presented the working way generated by the bucket cylinder, so the digging force generated by the bucket cylinder is:

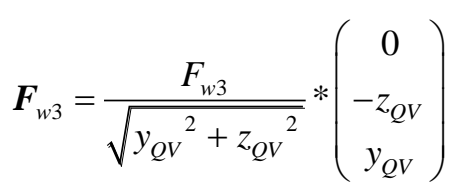

\section{The Result of the Static Force at Point $K$ in Three Dimensions}

Substituting equation 38 into equations 22 and 23, the magnitudes of the unknown forces $\boldsymbol{F}_{K y}$ and $\boldsymbol{F}_{K z}$ can be calculated:

$$
\begin{gathered}
F_{K y}=\frac{\left(-F_{w 3} \sqrt{y_{Q V}{ }^{2}+z_{Q V}{ }^{2}}+\sum_{n=10}^{13} G_{i} y_{Q i}\right) y_{H K}+G_{9} y_{H 9} y_{Q K}}{z_{H K} y_{Q K}-z_{Q K} y_{H K}} \\
F_{K z}=\frac{\left(-F_{w 3} \sqrt{\left.{y_{Q V}{ }^{2}+z_{Q V}{ }^{2}}^{2}+\sum_{n=10}^{13} G_{i} y_{Q i}\right) z_{H K}+G_{9} y_{H 9} z_{Q K}}\right.}{z_{H K} y_{Q K}-z_{Q K} y_{H K}}
\end{gathered}
$$

\section{The Results of the Static Force at Point K in Two Dimensions}

According to [1], the result of the component force at point $\mathrm{K}$ in the direction of perpendicular to the line QK is:

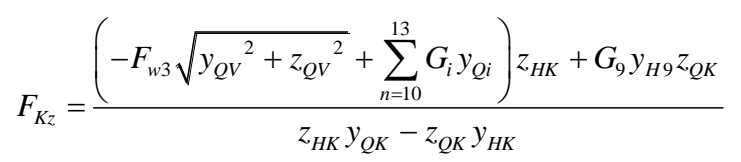


Where $l_{3}$ is the distances between the Point $\mathrm{Q}$ and $\mathrm{V}, l_{3}=\sqrt{{y_{Q V}{ }^{2}+z_{Q V}{ }^{2}}^{2}} ; l_{Q K}$ is the distances between the Point $\mathrm{Q}$ and $\mathrm{K}, l_{Q K}=\sqrt{{y_{Q K}{ }^{2}+z_{Q K}{ }^{2}}^{2}}$; According to the reference [1], $\mathrm{G}_{10}, \mathrm{G}_{11}$ should be a negative value.

According to [1], the result of the component force at point $\mathrm{K}$ in the direction of line $\mathrm{QK}$ is:

$$
F_{K u}=\frac{F_{K v}\left(y_{H K} \cos \varphi_{Q K}+z_{H K} \sin \varphi_{Q K}\right)-G_{9} y_{H 9}}{z_{H K} \cos \varphi_{Q K}-y_{H K} \sin \varphi_{Q K}}
$$

Where $\varphi_{Q K}$ is the angle between the axis and vector which is the relative position vector of the

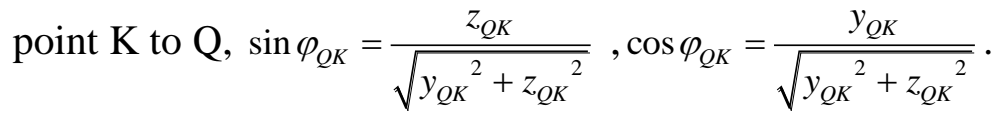

According to [1], the transformation matrix of the result of the force at point $\mathrm{K}$ from the local coordinates to global coordinates is:

$$
\left(\begin{array}{c}
F_{K x} \\
F_{K y} \\
F_{K z}
\end{array}\right)=\left(\begin{array}{c}
0 \\
F_{K u} \cos \varphi_{Q K}-F_{K \nu} \sin \varphi_{Q K} \\
F_{K u} \sin \varphi_{Q K}+F_{K \nu} \cos \varphi_{Q K}
\end{array}\right)
$$

Substituting equations 41 and 42 into equation 43 yields:

$$
\begin{gathered}
F_{K y}=\frac{F_{K v} y_{H K}-G_{9} y_{H 9} \cos \varphi_{Q K}}{z_{H K} \cos \varphi_{Q K}-y_{H K} \sin \varphi_{Q K}} \\
F_{K z}=\frac{F_{K v} z_{H K}-G_{9} y_{H 9} \sin \varphi_{Q K}}{z_{H K} \cos \varphi_{Q K}-y_{H K} \sin \varphi_{Q K}}
\end{gathered}
$$

Substituting $l_{3}=\sqrt{{y_{Q V}{ }^{2}+z_{Q V}{ }^{2}}^{2}}, l_{Q K}=\sqrt{{y_{Q K}{ }^{2}+z_{Q K}{ }^{2}}^{2}}, \sin \varphi_{Q K}=\frac{z_{Q K}}{\sqrt{y_{Q K}{ }^{2}+z_{Q K}{ }^{2}}}, \cos \varphi_{Q K}==\frac{y_{Q K}}{\sqrt{y_{Q K}{ }^{2}+z_{Q K}{ }^{2}}}$ into equations 44 and 45, the magnitudes of the unknown forces $\boldsymbol{F}_{K y}$ and $\boldsymbol{F}_{K z}$ can be calculated:

$$
\begin{gathered}
F_{K y}=\frac{-\left(F_{w 3} \sqrt{y_{Q V}{ }^{2}+z_{Q V}{ }^{2}}+G_{10} y_{Q 10}+G_{11} y_{Q 11}\right) y_{H K}-G_{9} y_{H 9} y_{Q K}}{z_{H K} y_{Q K}-z_{Q K} y_{H K}} \\
F_{K z}=\frac{-\left(F_{w 3} \sqrt{\left.{y_{Q V}{ }^{2}+z_{Q V}{ }^{2}}^{2}+G_{10} y_{Q 10}+G_{11} y_{Q 11}\right) z_{H K}-G_{9} y_{H 9} z_{Q K}}\right.}{z_{H K} y_{Q K}-z_{Q K} y_{H K}}
\end{gathered}
$$

\begin{tabular}{|c|c|c|c|}
\hline $\begin{array}{c}\text { Force } \\
\text { at } \\
\text { point } \\
\mathrm{K} \\
\end{array}$ & $\begin{array}{c}\text { In two dimension } \\
\text { (according to the reference, } \mathrm{G}_{9}, \mathrm{G}_{10} \text { and } \\
\mathrm{G}_{10} \text { should be a negative value) }\end{array}$ & In three dimension & $\begin{array}{c}\text { Introducti } \\
\text { ons }\end{array}$ \\
\hline \multirow{2}{*}{$F_{K y}$} & $-\left(F_{w 3} \sqrt{y_{Q V}^{2}+z_{Q V}^{2}}+G_{10} y_{Q 10}+G_{11} y_{Q 11}\right) y_{H K}-G_{9} y_{H 9} y_{Q K}$ & $\left(-F_{w 3} \sqrt{y_{Q V}{ }^{2}+z_{Q V}{ }^{2}}+\sum_{n=10}^{13} G_{i} y_{Q i}\right) y_{H K}+G_{9} y_{H 9} y_{Q K}$ & \multirow{4}{*}{$\begin{array}{l}\sum_{n=10}^{13} G_{i} y_{Q i} \\
\text { considered } \\
\text { the gravity } \\
\text { of the } \\
\text { tiltrotator }\end{array}$} \\
\hline & $z_{H K} y_{Q K}-z_{Q K} y_{H K}$ & $z_{H K} y_{Q K}-z_{Q K} y_{H K}$ & \\
\hline \multirow{2}{*}{$F_{K z}$} & $-\left(F_{w 3} \sqrt{y_{Q V}^{2}+z_{Q V}^{2}}+G_{10} y_{Q 10}+G_{11} y_{Q 11}\right) z_{H K}-G_{9} y_{H 9} z_{Q K}$ & 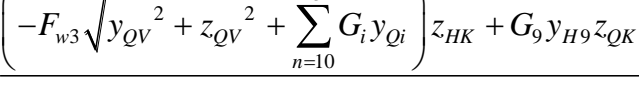 & \\
\hline & $z_{H K} y_{Q K}-z_{Q K} y_{H K}$ & $z_{H K} y_{Q K}-z_{Q K} y_{H K}$ & \\
\hline
\end{tabular}

Where according to the reference [1], $\mathrm{G}_{9}, \mathrm{G}_{10}$ and $\mathrm{G}_{11}$ should be a negative value.

Table 1. Comparison of the force at point $\mathrm{K}$ 
Table 1 shows the comparison to the force analysis result at the point $\mathrm{K}$. The one result is determined by the method presented in this paper, and the other is determined by the classical method. From the table 1, the planar force results can be compatible with spatial calculation result at point $\mathrm{K}$. The gravity of tiltrotator is considered in the calculation of the spatial force analysis. Without taking into account the structure, namely the excavator without a tiltrotator, the forces at point $\mathrm{K}$ calculated by two different methods are the same.

\section{Comparison of the Force at Point $Q$}

\section{The Result of the Static Force at Point $K$ in Three Dimensions}

Spatial force results at point $\mathrm{Q}$ are equations $34 \sim 37$, substituting equation 38 into equations $34 \sim 37$, the unknown forces at $Q$ point can be calculated:

$$
\begin{gathered}
F_{Q z}=-\left(F_{w 3 z}+F_{K z}-\sum_{n=10}^{13} G_{i}\right) \\
F_{Q y}=-\left(F_{K y}+F_{w 3 y}\right) \\
F_{Q x}=0 \\
M_{Q \mathrm{y}}=F_{w 3 z} x_{Q V}-\sum_{n=10}^{13} G_{i} x_{Q i} \\
M_{Q z}=F_{w 3 y} x_{Q V}
\end{gathered}
$$

\section{The Results of the Static Force at Point $Q$ in Two Dimensions}

According to [1], planar force results at point Q are:

$$
F_{Q z}=-F_{w 3 z}-F_{K z}-G_{10}-G_{11}
$$

\begin{tabular}{|c|c|c|c|}
\hline $\begin{array}{l}\text { Force } \\
\text { at point } \\
\text { Q }\end{array}$ & $\begin{array}{c}\text { In two dimension } \\
\text { (according to the reference, } \mathrm{G}_{10} \text { and } \\
\mathrm{G}_{11} \text { should be a negative value) }\end{array}$ & In three dimension & Introductions \\
\hline$F_{Q z}$ & $-F_{w 3 z}-F_{K z}-G_{10}-G_{11}$ & $-\left(F_{w 3 z}+F_{K z}-\sum_{n=10}^{13} G_{i}\right)$ & \multirow{2}{*}{$\begin{array}{l}\sum_{n=10}^{13} G_{i} y_{Q i} \text { considered the } \\
\text { gravity of the tiltrotator }\end{array}$} \\
\hline$F_{Q y}$ & $-F_{K y}-F_{w 3 y}$ & $-\left(F_{K y}+F_{w 3 y}\right)$ & \\
\hline$F_{Q x}$ & & 0 & \multirow{3}{*}{$\begin{array}{l}\text { These are generated by } \\
\text { considering the bucket } \\
\text { deviate from the } \\
\text { working plane }\end{array}$} \\
\hline$M_{Q \mathrm{y}}$ & & $F_{w 3 z} x_{Q V}-\sum_{n=10}^{13} G_{i} x_{Q i}$ & \\
\hline$M_{Q z}$ & & $F_{w 3 y} x_{Q V}$ & \\
\hline
\end{tabular}

Where according to the reference [1], G10, G11 should be a negative value.

$$
F_{Q y}=-F_{K y}-F_{w 3 y}
$$

Table 2. Comparison of the force at point $\mathrm{Q}$

Table 2 shows the comparison of the force analysis result at the point Q. The one result is determined by the method presented in this paper, and the other is determined by the classical method. From the table 2, the planar force results can be compatible with spatial calculation result at the point Q. Three dimensional calculation results are to consider a bucket deviate from the plane of symmetry of the excavator attachment, so there are added moments of the forces about $\mathrm{Y}$ and $\mathrm{Z}$-axis. If the deviating of bucket had not been considered, namely the excavator without a tiltrotator, the 
corresponding added moments could not have existed. In this condition, the forces at the point Q calculated by two different methods are the same.

Three other groups can also be analysed by using the calculation method of the first group, as its results are easy to prove the compatibility to the calculation of the plane. The static force analysis performed by considering the tiltrotator can be used as a boundary condition for static FEA of the excavator attachments [8].

\section{Conclusion}

The calculation procedures presented in this paper can be applied to the parts of the excavator attachments to calculate the reaction forces at the hinge points accurately. Especially these calculation procedures can be used to solve the reaction force of the excavator attachments with tiltrotator that are acted by a spatial force system.

According to the structure of the excavator attachments and characteristics of the force, the excavator attachments can be divided into 4 linkage groups. The force can be calculated by one of the groups, and the forces of other groups can be deduced. In this way, the force analysis of the excavator attachments is simplified.

By comparing the results of the force analysis, which calculated by the two methods, the compatibility and correctness of the method presented in this paper have been verified. The static force analysis of the excavator with tiltrotator can be used as a boundary condition for static FEA of the excavator attachments.

\section{References}

1. Qinglu Shi, China machine press, Hydraulic excavator, (2011)

2. Chunli Liu, Master Thesis from Northeaster University, Structure analysis and optimum design of excavator quick change device, (2013)

3. Tongji University, Beijing: China architecture and building press. Single bucket hydraulic excavator, (1986)

4. Shangnian Jin, Ma. Higher Education Press, Theoretical mechanics, (2002)

5. Yongxu Li, Master Thesis from Tongji University, Hydraulic system design of hydraulic excavator working equipment and research, (2006)

6. M. babu, International Journal of Engineering Research and Applications, Static Force Analysis of Mini Hydraulic Backhoe Excavator And Evaluation Of Bucket Capacity, Digging Force Calculations, 25-32 (2015)

7. P. Patel, J. M. Prajapati, J. Gadhvi, International Journal of Engineering Science \& Technology, An Excavation Force Calculations and Applications: Analytical Approach, 3, 3831-3837 (2011)

8. Z. Towarek, International Journal of Mechanical Sciences, Dynamics of a single-bucket excavator on a deformable soil foundation during the digging of ground, 45, 1053-1076 (2003) 